Ton J. Cleophas · Aeilko H. Zwinderman

\title{
Machine Learning in Medicine - Cookbook
}

Springer 


\section{Contents}

\section{Part I Cluster Models}

1 Hierarchical Clustering and K-means Clustering to Identify

Subgroups in Surveys $(50$ Patients) . . . . . . . . . . . . 3

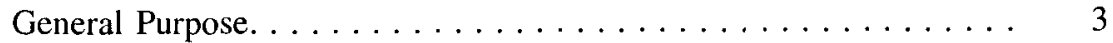

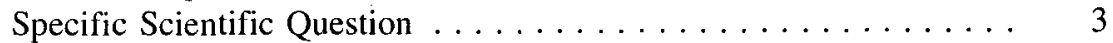

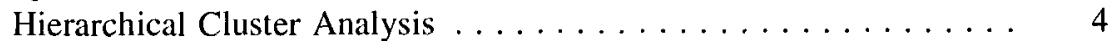

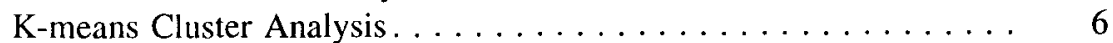

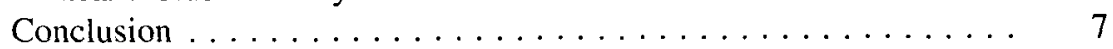

Note. . . . . . . . . . . . . . . . . . . . . 8

2 Density-Based Clustering to Identify Outlier Groups

in Otherwise Homogeneous Data (50 Patients) . . . . . . . . 9

General Purpose. . . . . . . . . . . . . . . . . . . 9

Specific Scientific Question .................... 9

Density-Based Cluster Analysis . . . . . . . . . . . . . . 10

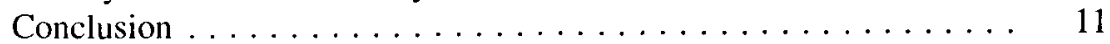

Note. . . . . . . . . . . . . . . . . . . . . . . . . 11

3 Two Step Clustering to Identify Subgroups and Predict

Subgroup Memberships in Individual Future

Patients (120 Patients) $\ldots \ldots \ldots \ldots \ldots \ldots \ldots \ldots \ldots$

General Purpose. . . . . . . . . . . . . . . . . . . . 13

Specific Scientific Question .................... 13

The Computer Teaches Itself to Make Predictions . . . . . . . . . . 14

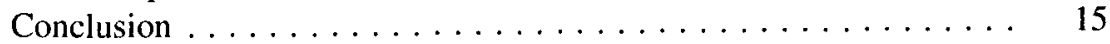

Note . . . . . . . . . . . . . . . . . . . . . . 15

\section{Part II Linear Models}

4 Linear, Logistic, and Cox Regression for Outcome Prediction with Unpaired Data (20, 55, and 60 Patients) . . . . . . . . . 19

General Purpose. ........................ 19 
Specific Scientific Question . . . . . . . . . . . . . . . . . . 19

Linear Regression, the Computer Teaches Itself

to Make Predictions . . . . . . . . . . . . . . . . . . . . . . 19

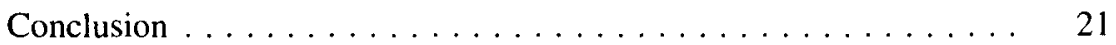

Note . . . . . . . . . . . . . . . . . . . . . . . 21

Logistic Regression, the Computer Teaches Itself

to Make Predictions . . . . . . . . . . . . . . . . . . 22

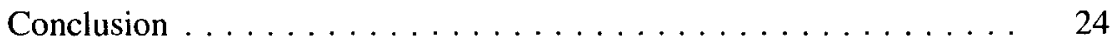

Note. . . . . . . . . . . . . . . . . . . . . . 24

Cox Regression, the Computer Teaches Itself

to Make Predictions . . . . . . . . . . . . . . . . . . . . 24

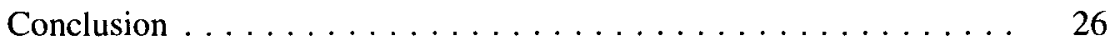

Note . . . . . . . . . . . . . . . . . . . . . . 26

5 Generalized Linear Models for Outcome Prediction

with Paired Data (100 Patients and 139 Physicians) . . . . . . . . . 29

General Purpose. . . . . . . . . . . . . . . . . . . . . . . . 29

Specific Scientific Question . . . . . . . . . . . . . . . . . . 29

Generalized Linear Modeling, the Computer Teaches Itself

to Make Predictions . . . . . . . . . . . . . . . . . . . . . . . . 29

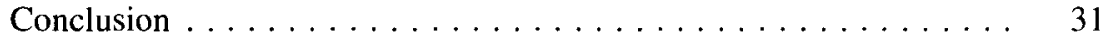

Generalized Estimation Equations, the Computer Teaches Itself

to Make Predictions . . . . . . . . . . . . . . . . . . 32

Conclusion ........................ 34

Note. . . . . . . . . . . . . . . . . . . . . . . . . . . 35

6 Generalized Linear Models for Predicting

Event-Rates (50 Patients) . . . . . . . . . . . . . . . . . . 37

General Purpose. . . . . . . . . . . . . . . . . . . . . . . . 37

Specific Scientific Question . . . . . . . . . . . . . . . . 37

The Computer Teaches Itself to Make Predictions . . . . . . . . . 38

Conclusion . . . . . . . . . . . . . . . . . . . . . . . 40

Note . . . . . . . . . . . . . . . . . . . . . . . . . . . 41

7 Factor Analysis and Partial Least Squares for Complex-Data

Reduction (250 Patients) . . . . . . . . . . . . . . . . . 43

General Purpose. . . . . . . . . . . . . . . . . . . . . 43

Specific Scientific Question ... . . . . . . . . . . . . . 43

Factor Analysis . . . . . . . . . . . . . . . . . . . 44

Partial Least Squares Analysis. . . . . . . . . . . . . . . . . . . 46

Traditional Linear Regression . . . . . . . . . . . . . . . . . . 48

Conclusion .......................... 48

Note. . . . . . . . . . . . . . . . . . . . . . . . . . 49 
8 Optimal Scaling of High-Sensitivity Analysis of Health

Predictors $(250$ Patients) $\ldots \ldots \ldots \ldots \ldots \ldots \ldots$

General Purpose. . . . . . . . . . . . . . . . . . . 51

Specific Scientific Question .................. 51

Traditional Multiple Linear Regression. . . . . . . . . . . . . 52

Optimal Scaling Without Regularization. . . . . . . . . . . 53

Optimal Scaling with Ridge Regression . . . . . . . . . . . . . . 54

Optimal Scaling with Lasso Regression . . . . . . . . . . . . . 54

Optimal Scaling with Elastic Net Regression. . . . . . . . . . . 55

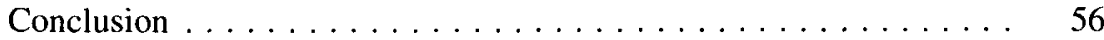

Note . . . . . . . . . . . . . . . . . . . . . . . . . . 56

9 Discriminant Analysis for Making a Diagnosis from Multiple

Outcomes (45 Patients) . . . . . . . . . . . . . . . 57

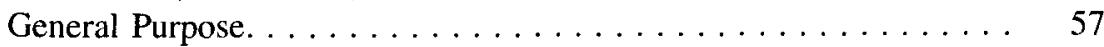

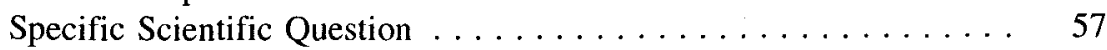

The Computer Teaches Itself to Make Predictions . . . . . . . . . 58

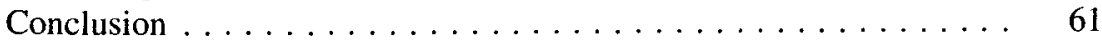

Note . . . . . . . . . . . . . . . . . . . . . . . . . 61

10 Weighted Least Squares for Adjusting Efficacy Data

with Inconsistent Spread $(78$ Patients) $\ldots \ldots \ldots \ldots \ldots 63$

General Purpose. . . . . . . . . . . . . . . . . . . 63

Specific Scientific Question . . . . . . . . . . . . . . . . . . 63

Weighted Least Squares . . . . . . . . . . . . . . . . . 64

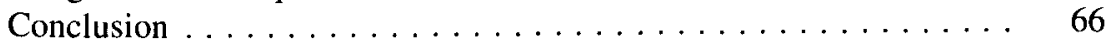

Note . . . . . . . . . . . . . . . . . . . . . . 66

11 Partial Correlations for Removing Interaction Effects

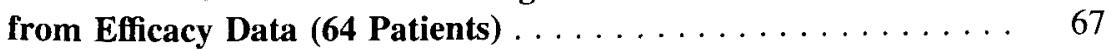

General Purpose. . . . . . . . . . . . . . . . . . . . 67

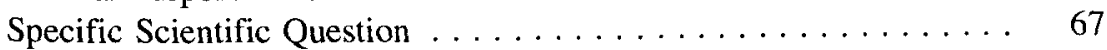

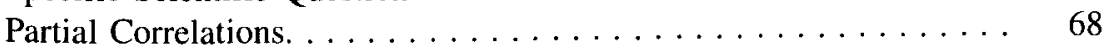

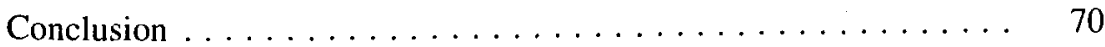

Note . . . . . . . . . . . . . . . . . . . . . . 71

12 Canonical Regression for Overall Statistics of Multivariate

Data $(250$ Patients) . . . . . . . . . . . . . . . . . . . . . 73

General Purpose. . . . . . . . . . . . . . . . . . . . . 73

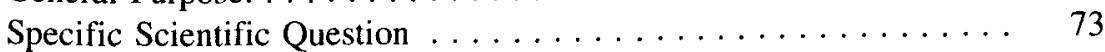

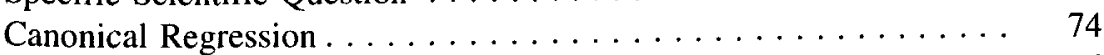

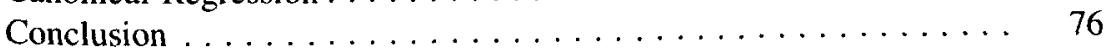

Note. . . . . . . . . . . . . . . . . . . . 77 


\section{Part III Rules Models}

13 Neural Networks for Assessing Relationships that are Typically Nonlinear $(90$ Patients $) \ldots \ldots \ldots 1 \ldots \ldots$

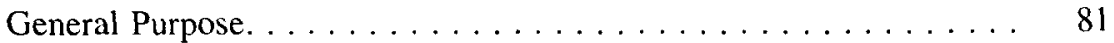

Specific Scientific Question ................... 81

The Computer Teaches Itself to Make Predictions . . . . . . . . . . 82

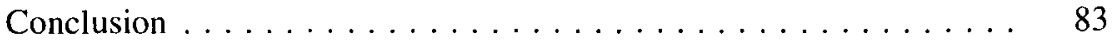

Note . . . . . . . . . . . . . . . . . . . . . . . . . 83

14 Complex Samples Methodologies for Unbiased

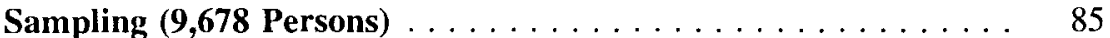

General Purpose. . . . . . . . . . . . . . . . . . . . 85

Specific Scientific Question . . . . . . . . . . . . . . 85

The Computer Teaches Itself to Predict Current Health Scores

from Previous Health Scores . . . . . . . . . . . . . . . 87

The Computer Teaches Itself to Predict Odds Ratios

of Current Health Scores Versus Previous Health Scores . . . . . . . 88

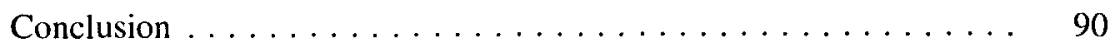

Note. . . . . . . . . . . . . . . . . . . 90

15 Correspondence Analysis for Identifying the Best of Multiple Treatments in Multiple Groups (217 Patients) . . . . . . . . . . 91

General Purpose. . . . . . . . . . . . . . . . . . . . 91

Specific Scientific Question . . . . . . . . . . . . . . . . 91

Correspondence Analysis . . . . . . . . . . . . . . . . . . 92

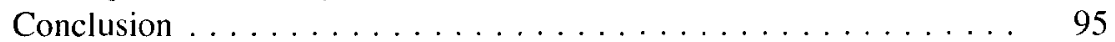

Note . . . . . . . . . . . . . . . . . . . . . . . 95

16 Decision Trees for Decision Analysis (1,004 and 953 Patients) ... 97

General Purpose. . . . . . . . . . . . . . . . . . . . . . . . 97

Specific Scientific Question . . . . . . . . . . . . . . . . . . 97

Decision Trees with a Binary Outcome . . . . . . . . . . . . . 97

Decision Trees with a Continuous Outcome . . . . . . . . . . 101

Conclusion ... . . . . . . . . . . . . . . . . . . . . . . . 104

Note . . . . . . . . . . . . . . . . . . . . . . . . . . . . . 104

17 Multidimensional Scaling for Visualizing Experienced Drug

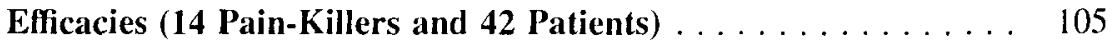

General Purpose. . . . . . . . . . . . . . . . . . . . . 105

Specific Scientific Question . . . . . . . . . . . . . . . . 105

Proximity Scaling . . . . . . . . . . . . . . . . . . . . . . . . 105

Preference Scaling . . . . . . . . . . . . . . . . . . . 108

Conclusion .......................... 112

Note. . . . . . . . . . . . . . . . . . . . . . . . . . . . . . . 113 
18 Stochastic Processes for Long Term Predictions

from Short Term Observations . . . . . . . . . . . . . . . 115

General Purpose. . . . . . . . . . . . . . . . . . 115

Specific Scientific Questions . . . . . . . . . . . . . . . . 115

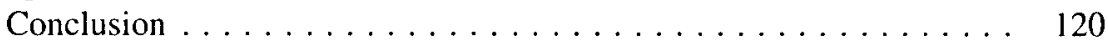

Note. . . . . . . . . . . . . . . . . . . . . . 121

19 Optimal Binning for Finding High Risk

Cut-offs $(1,445$ Families) . . . . . . . . . . . . . . . . . . . . 123

General Purpose. . . . . . . . . . . . . . . . . . . . 123

Specific Scientific Question . . . . . . . . . . . . . . . 123

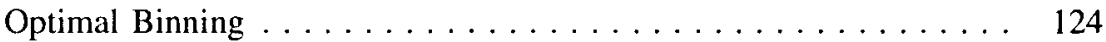

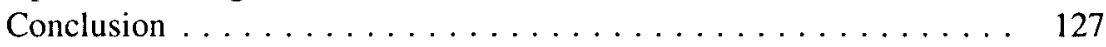

Note. . . . . . . . . . . . . . . . . . . . . . 127

20 Conjoint Analysis for Determining the Most Appreciated

Properties of Medicines to be Developed (15 Physicians) . . . . . . 129

General Purpose. . . . . . . . . . . . . . . . . . . . . . . . . 129

Specific Scientific Question . . . . . . . . . . . . . . . . . . . 129

Constructing an Analysis Plan. . . . . . . . . . . . . . . . 129

Performing the Final Analysis . . . . . . . . . . . . . . 131

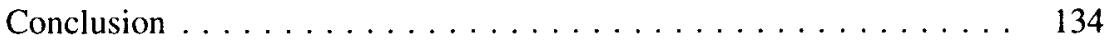

Note. . . . . . . . . . . . . . . . . . . . . . . . . . . . . . 134

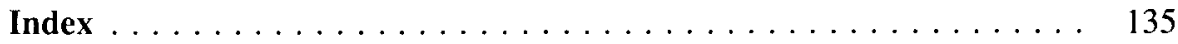

